\title{
Simple rehearsal strategies for short-term recall'
}

MURIEL $M$. WOODHEAD

APPLIED PSYCHOLOGY RESEARCH UNIT, CAMBRIDGE, ENGLAND

Four separate groups of $S s$ were assigned differing rehearsal strategies by which to memorize the randomized words of a sentence in the orders presented. Ss made less errors in recall after vocal rehearsal than after non-vocal. Reading the words aloud twice without increasing the presentation time did not appear to delay the decay of the memory trace. Duplicated vocal and silent rehearsals appeared to have undefined intermediate roles.

During the practice runs of a difficult task of continuing memory (Woodhead, 1966), many Ss had to be discouraged from rehearsing aloud. The present study was made to discover whether vocalizing was more efficient than silent rehearsal. At this time the study of vocalization in short-term memory by Murray (1966) had not appeared. Murray used a rather complex 2 by 2 by 2 by 2 by 4 factorial design, by which all of his Ss read items vocally and silently, spoke and wrote answers, were and were not informed of the type of response required, recalled to "free" and "forced" instruction, and perceived at four rates of presentation. His Ss were recalling eight of 16 consonants and they performed best after vocalizing during fast presentation. Murray suggested that storage is facilitated by voicing because interference is resisted and, possibly, decay is delayed.

The sensitivity of experiments in which Ss perform every condition can be reduced if there are any asymmetrical transfer effects between conditions (Poulton, 1966). It seems useful to study short-term memorizing when each $\mathrm{S}$ meets only one condition. The advantage of vocalization should be marked even when separate groups of Ss are used. Murray also found that the stimuli could be acoustically confused with one another. This further variable can be avoided by substituting a fixed set of items, so that the task is simply to reproduce them in the correct order. A simple design of this nature was chosen for the present study of shortterm recall.

The following predictions were made: (1) With a fast rate of presentation of items, short-term storage would be facilitated by rehearsing aloud (vocal rehearsal being the preferred strategy in the continuing memory study mentioned above). (2) Given the same short exposure time, rehearsing by reading the items aloud twice would be a less effective way of learning; in fitting the critical time limit, the first reading may be a distraction from the attempt to remember the order of the items, while approximately a half only of the total exposure time is left for the second vocalization, from which the order must be recalled. A lack of gain in performance from the vocal repetition of identical items without extension of time, would indicate one exception t the proposition that voicing facilitates storage and perhaps delays the decay of a trace. (3) Given the same presentation rate, the time stress underlying the second prediction may not hold when the double rehearsal is silent; this condition need not be inferior and could be superior to single silent rehearsal if the time spared from nonvoicing is spent on "strengthening" the trace, either during a brief pause between the two silent readings, or by reading each word more slowly. Tentatively, the order of efficiency predicted for rehearsing under the stress of brief visual presentation was that single vocal rehearsal would be superior, followed by double vocal rehearsal, then double silent rehearsal equalled, or followed, by single silent rehearsal.

Method

The Ss tested individually, were 48 housewives whose ages ranged from 22-57. They were matched by age to form four comparable groups. Each group was assigned a different memorizing strategy.

The stimulus material was formed from a sentence, "how few can win yet all may try," which provides eight common three-letter words with differing initial letters and vowel sounds. Twenty-four randomized versions of the eight words were constructed. A version was exposed for $4 \mathrm{sec}$, after which the $\mathrm{S}$ wrote the eight words in any order, as long as they appeared on the answer sheet in the serial order presented, and without omissions. In preliminary practice, the Ss were guided towards making full use of the $4 \mathrm{sec}$. for their respective rehearsal strategies. These were: reading the words aloud once, reading aloud twice, reading silently once, reading silently twice. The silent groups were required to run a pen under the words to ensure the number of times of reading. Four sec. appeared to be the minimum time in which pilot Ss could or would consistently read 16 randomized words aloud, although they could read the original non-random version more rapidly.

\section{Results and Discussion}

Given the predicted order of value of alternative rehearsals, the error scores were tested by Jonckheere's test for independent samples, which gave $\mathrm{z}=3.95, \mathrm{p}<.001$. This result indicates that it was easiest to recall the words in the correct order of presentation after a single vocal rehearsal (24\% errors), rather less easy with double vocal rehearsal $(29 \%$ errors), worse with double silent rehearsal $(43 \%$ errors), and worst with single silent rehearsal $(45 \%$ errors). 


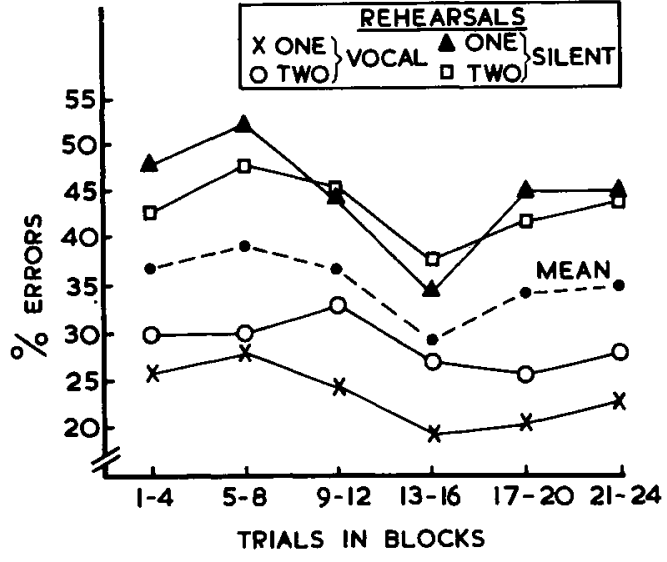

Fig. 1. Errors in recall made by four groups of $12 \mathrm{Ss}$ assigned differing rehearsal strategies. Each point indicates \% errors on four trials by $12 \mathrm{Ss}$.

The distribution of errors over blocks of trials of the randomized word versions appears in Fig. 1. The performance curves differed from each other at each of the 24 trials, by the Mann-Whitney $U$ statistic $(p<.01$ ), with the exception of the single and double silent rehearsals. Comparisons of the total errors of each $S$ revealed that vocal was superior to silent rehearsal $(p=.003)$ and also to double silent rehearsal $(p=.004)$, that double vocal rehearsal was superior to silent rehearsal $(p=.006)$ and to double silent rehearsal $(p=.016)$. One possible reason for the smaller probable level of significance in the latter comparison could be the stress of the very rapid vocalizing necessary to articulate the randomized words twice. There was an insignificant tendency for single vocal rehearsal to be better than double vocal rehearsal, but no difference between the two silent conditions.

The results fully support Murray's finding that reading aloud improves short-term recall of eight items. In the present instance, no advantage was gained by vocalizing twice, perhaps because the time was too short or because the second vocal reading merely duplicated the auditory input, while the first at least provided an auditory as well as visual input. The four conditions of rehearsal do form a rank order, suggesting the existence of intermediate stages, between vocal and non-vocal, of rehearsal values for recall. Finally, although extra vocalizing was not beneficial, vocalizing per se was an advantage.

\section{References}

Murray, D. J. Vocalization-at-presentation and immediate recall, with varying recall methods. Quart. J. exp. Psychol., 1966, 8, 9-18.

Poulton, E. C. Engineering Psychology. Annu. Rev. Psychol., $1966,17,177-200$

Woodhead, M. M. Varying the number of alternatives in short-term recall. Brit. J. Psychol., 1966, 57, 45-52.

\section{Note}

1. This research was supported by the British Medical Research Council. 